ProbeTec lysis buffer. US and UPT urine specimens were processed according to the manufacturer's recommended procedure with the BD ProbeTec ET assay (Becton Dickinson).

Results Out of 134 males from the detention center and the STD clinic, 115 were negative and 19 positive for either CT or NG in both US and UPT collection devices. Five patients were positive for both CT and GC. The CT prevalence was $8.2 \%$. The NG prevalence was $10.44 \%$.

Conclusions Good results agreement was found between the Copan UriSwab and the BD urine collection devices for the detection of CT and GC with the BD ProbeTec ET assay. The UriSwab is easy to transport and process in the laboratory for the detection of CT, GC and other STI infectious agents with molecular assays and can also be used for culturing all urogenital bacteria. The UriSwab can facilitate self-collection for STI screening.

\section{P3-S1.32 A VALIDATION STUDY OF THE GEN-PROBE APTIMA COMB02 (AC2) ASSAY FOR DETECTING CHLAMYDIA TRACHOMATIS AND NEISSERIA GONORRHOEAE IN DRY SWABS}

doi:10.1136/sextrans-2011-050108.432

${ }^{1} \mathrm{~L}$ Cosentino, ${ }^{2} \mathrm{~J} \mathrm{R}$ Schwebke, ${ }^{3} \mathrm{M}$ M Hobbs, ${ }^{4} \mathrm{~S} \mathrm{~L}$ Hillier. ${ }^{1}$ Magee Womens Research Institute, Pittsburgh, USA; ${ }^{2}$ University of Alabama, Birmingham, USA; ${ }^{3}$ University of North Carolina, Chapel Hill, USA; ${ }^{4}$ University of Pittsburgh, Magee Womens Research Institute, Pittsburgh, USA

Background Vaginal swabs are an optimal specimen for detection of Chlamydia trachomatis (CT) and Neisseria gonorrhoeae (GC). The GenProbe Aptima system (Aptima) requires use of a sample transport media, whereas the Becton Dickinson ProbeTec ET System (Probetec) can utilise dry swabs. The use of dry swabs allows for collection of STD test samples at non-clinical testing venues.

Methods Swabs were collected from 180 sexually active women aged 15-25 years, who had asymptomatic BV and two or more risk factors for STDs. The participants received home vaginal swab self-collection kits for BV and STD testing. Participants mailed the kits directly to the lab. Probetec swabs were tested within 14 days of collection. A dry transport swab was placed into an Aptima vaginal swab collection tube, generally within 14 days of collection and stored at $-80^{\circ} \mathrm{C}$. Aptima swabs were thawed and tested in batches. Specimens with discordant results in the two nucleic acid amplification test systems were retested with both systems.

Results There were 58 women (32\%) positive for GC and 62 (34\%) positive for CT. The level of agreement between the Aptima and Probetec systems was higher for CT (176/180, 98\%) than for GC (171/180, 95\%). Of the 13 samples with discordant results, five were resolved with repeat testing. All eight remaining samples had discordant GC results: seven were Probetec positive, Aptima negative and one was Probetec negative, Aptima positive.

Conclusions Vaginal swabs tested in the Aptima system were equivalent to Probetec in detecting CT but were less sensitive for the detection of GC. Dry swabs cannot be recommended for detection of GC from vaginal swabs using the Aptima system.

\section{P3-S1.33 EVALUATION OF THE ROCHE COBAS 4800 FOR THE DETECTION OF CHLAMYDIA TRACHOMATIS AND NEISSERIA GONORRHOEAE USING MINIMALLY INVASIVE SAMPLES IN WOMEN}

doi:10.1136/sextrans-2011-050108.433

${ }^{1} \mathrm{~L}$ Hires, ${ }^{1} \mathrm{~B}$ Van Der Pol, ${ }^{2} \mathrm{~S}$ Taylor, ${ }^{2} \mathrm{R}$ Lillis, ${ }^{3} \mathrm{~B}$ Body. ${ }^{1}$ Indiana University School of Public Health, Bloomington, USA; ${ }^{2}$ Louisiana State University Health Sciences Center, New Orleans, USA; ${ }^{3}$ Laboratory Corporation of American, Burlington, USA

Background The Roche cobas 4800 (cobas) is a new diagnostic assay that utilises an automated workstation to isolate nucleic acids from clinical specimens and a real time instrument for the detection of $C$ trachomatis (CT) and $N$ gonorrhoeae (NG). The objective of this study was to compare the performance characteristics of the cobas to the BD Viper (Viper) and GenProbe Aptima Combo 2 (AC2) assays for the detection of CT and NG using a patient infected standard (PIS). Methods Specimens were obtained from women attending STD, family planning, or OB/GYN clinics from 12 geographically distinct locations. Urine and vaginal swabs were obtained from each participant as were endocervical (data not shown) and liquid based cytology samples (data not shown). Women were randomised to either self-obtained (SOV) or clinician-obtained (COV) vaginal swab collection. Four sites performed testing by AC2, cobas, and Viper for urine and by cobas for vaginal swabs. A patient was considered infected if at least two of the assays with different molecular targets gave positive results from cervical or urine samples.

Results Overall CT sensitivity ranged from $91.9 \%$ to $93.9 \%$ and specificity ranged from $99.7 \%$ to $99.8 \%$ for all sample types. Overall GC sensitivity ranged from $97.0 \%$ to $100 \%$ and specificity from 99.9\% to $100 \%$ see Abstract P3-S1.33 table 1 .

Conclusions The cobas assay has excellent sensitivity and specificity when compared to PIS. There was no difference in performance between the SOV and COV specimens or between the vaginal and urine specimens. Self-obtained vaginal swabs provide opportunities for increased efficiency within the clinical settings. The assay is easy to perform, automated, and can be completed in $<4 \mathrm{~h}$.

\section{P3-S1.34 EVALUATION OF THE ROCHE COBAS 4800 FOR THE DETECTION OF CHLAMYDIA TRACHOMATIS AND NEISSERIA GONORRHOEAE USING ENDOCERVICAL SPECIMENS}

doi:10.1136/sextrans-2011-050108.434

${ }^{1} \mathrm{~A}$ Pantone, ${ }^{1} \mathrm{~B}$ Van Der Pol, ${ }^{2} \mathrm{R}$ Lillis, ${ }^{3} \mathrm{~B}$ Loyd, ${ }^{3} \mathrm{M}$ B Nye, ${ }^{2} \mathrm{~S}$ Taylor. ${ }^{1}$ Indiana University School of Public Health, Bloomington, USA; ${ }^{2}$ Louisiana State University Health Sciences Center, New Orleans, USA; ${ }^{3}$ Laboratory Corporation of America, Burlington, USA

Background The Roche cobas 4800 (cobas) is a new diagnostic assay that utilises an automated workstation to isolate nucleic acids from clinical specimens and a real time instrument for the detection of $C$ trachomatis (CT) and $N$ gonorrhoeae (NG). The objective of this study was to compare the performance characteristics of the cobas to the BD Viper (Viper) and GenProbe Aptima Combo 2 (AC2) assays for the detection of CT and NG using a patient infected standard (PIS). Methods Specimens were obtained from women attending STD, family planning, or OB/GYN clinics from 12 geographically distinct

Abstract P3-S1.33 Table 1 Sample Type Vag

\begin{tabular}{|c|c|c|c|c|c|c|}
\hline Sample Type & $\mathbf{n}$ & CT Sensitivity [95\% CI] & CT Specificity [95\% Cl] & $\mathbf{n}$ & NG Sensitivity $[95 \%$ Cl] & NG Specificity $[95 \%$ Cl] \\
\hline Urine & 4271 & $92.3 \%(251 / 272)[88.5$ to 94.9$]$ & $\mathbf{9 9 . 7 \%}(3989 / 3999)$ [99.5 to 99.9$]$ & 4274 & $\mathbf{9 8 . 5 \%}(64 / 65)$ [91.8 to 99.7$]$ & $\mathbf{9 9 . 9 \%}(4206 / 4209)$ [99.8 to 100$]$ \\
\hline SOV & 2083 & $93.9 \%(123 / 131)[88.4$ to 96.9$]$ & $99.7 \%(1946 / 1952)$ [99.3 to 99.9$]$ & 2083 & $97.0 \%(32 / 33)[84.7$ to 99.5$]$ & $100 \%(2049 / 2050)$ [99.7 to 100$]$ \\
\hline $\mathrm{COV}$ & 2165 & $\mathbf{9 1 . 9 \%}(125 / 136)[86.1$ to 95.4$]$ & $99.8 \%(2024 / 20290)$ [99.4 to 99.9$]$ & 2164 & $100 \%(33 / 33)[89.6$ to 100.0$]$ & $100 \%(2130 / 2131)$ [99.7 to 100.0$]$ \\
\hline
\end{tabular}

